\title{
Biology and immature stages of the clam-killing fly, Renocera pallida (Diptera: Sciomyzidae)
}

\author{
JANA HORSÁKOVÁ \\ Department of Zoology and Ecology, Faculty of Science, Masaryk University, Kotlářská 2, 61137 Brno, Czech Republic; \\ e-mail: horsakova@email.cz
}

Key words. Sciomyzidae, Renocera pallida, clam-killing fly, rearing, life-cycle, egg, larva, puparium, morphology

\begin{abstract}
The larva of the Palaearctic Renocera pallida (Fallén, 1820) is confirmed as a predator of small species of bivalve molluscs of the family Sphaeriidae. To date only the larvae of three Nearctic Renocera species (and larvae of two other species of Sciomyzidae in two genera) are known to have the same food preference. The life cycle, biology, larval feeding and behaviour are described for the first time and compared with that of the Nearctic Renocera. The systematic position and biology of Renocera in general are discussed. Descriptions of the egg, second and third larval instars and the puparium of $R$. pallida are presented, the main features of the egg and larvae are illustrated by scanning electron micrographs.
\end{abstract}

\section{INTRODUCTION}

Since the first modern publication on the biology of larvae of Sciomyzidae by Berg (1953), the natural food and life cycles of about 200 species have been determined. Almost all the larvae reared are predators or parasitoids of terrestrial or aquatic gastropods, or consume snail eggs. Vala \& Gasc (1990) classified Sciomyzid larvae in to three categories according to their behavioural characteristics. The surface dwelling larvae (e.g., Dictya Meigen, 1803; Elgiva Meigen, 1838; Sepedon Latreille, 1804; some species of Ilione Haliday, 1837 and Tetanocera Duméril, 1800) are typical predators of pulmonate gastropods of the families Lymnaeidae, Planorbidae and Physidae. Larvae of the Palaearctic Ilione lineata (Fallén, 1820), Eulimnia philpotti Tonnoir et Malloch, 1928 from New Zealand and probably all species of Renocera Hendel, 1900 attack sphaeriid clams (Veneroida: Sphaeriidae) (Knutson \& Berg, 1967; Foote \& Knutson, 1970; Foote, 1976; Berg \& Knutson, 1978; Barnes, 1980). In the group of semi-aquatic predators and parasitoids (e.g., Colobaea Zetterstedt, 1837; Pteromicra Lioy, 1864; Sciomyza Fallén, 1820; many species of Pherbellia Robineau-Desvoidy, 1830) of hydrophilous and amphibious snails are included larvae of, e.g., Pherbina Robineau-Desvoidy, 1830; Psacadina Enderlein, 1939; Hydromya Robineau-Desvoidy, 1830 and some Tetanocera spp. that show parasitoid tendencies and are typical inhabitants of sheltered shore lines (Verbeke \& Knutson, 1967; Knutson et al., 1975; Rozkošný, 1997, 1998). A few species have very specialized habits. All larvae of Anticheta Haliday, 1838 utilize only exposed egg masses of snails of the genera Lymnaea Lamarck, 1799 and Succinea Draparnaud, 1801 (Fisher \& Orth, 1964; Knutson, 1966; Knutson \& Abercrombie, 1977; Robinson \& Foote, 1978). The species with terrestrial larvae are associated only with terrestrial gastropods. Tetanocera clara Loew, 1862; T. plebeja Loew, 1862 and
T. valida Loew, 1862 in North America (Trelka \& Foote, 1970); T. elata (Fabricius, 1781) and perhaps also Euthycera chaerophylli (Fabricius, 1798) in the Palaearctic (Knutson et al., 1965) are specialized parasitoids/predators on various slugs. Apparently only the primitive Salticella fasciata (Meigen, 1830), is able to feed on various dead invertebrates in addition to living terrestrial snails (Knutson et al., 1970; Coupland et al., 1994). The most recent data show that $S$. fasciata is not the only species that utilizes other groups of invertebrates as an additional or exclusive food source. Vala et al. (2000) published the first information on the biology of the Afrotropical Sepedonella nana Verbeke, 1950, the larvae of which are specialized predators of the freshwater Aulophorus furcatus (O.F. Müller, 1773) (Annelida: Oligochaeta: Naididae).

\section{Systematic position of Renocera}

The genus may be characterized as follows: Renocera resembles Tetanocera spp. in its shape and coloration, but on average it is distinctly smaller. One or two orbital setae, midfrontal stripe relatively narrow and shiny. Second antennal segment less than half the length of the third segment, its apex rounded, arista mostly plumose, with black hairs. Pro-episternum setose or bare, anepisternum and anepimeron without hairs, katepisternum fine haired, subalar setae absent, four scutellar setae. Dorsal inner margins of hind coxae bare. Ventral setae of hind femora rather strong in both sexes or reduced in females. Each hind tibia with one pre-apical seta. Wings without special pattern. Male sternite 5 with incision or two spinose tubercles. Surstyli simple in $R$. stroblii Hendel, 1900, and bipartite in the other two Palaearctic species (cf. Rozkošný, 1987).

On the basis of morphological studies of the postabdomen, including the male genitalia, Verbeke (1950) proposed the new subfamily Renocerinae for Renocera and Anticheta (incl. Hemitelopteryx Cresson, 1920). Steyskal 
(1965) and virtually all recent authors place Renocera close to Tetanocera in the tribe Tetanocerini of the subfamily Sciomyzinae. In the cladogram of Marinoni \& Mathis (2000), Renocera appears in a polytomy with Ectinocera Zetterstedt, 1838 at the base of the Tetanocerini clade. According to Foote (1976) larvae of Renocera in common with Anticheta (Fisher \& Orth, 1964; Knutson, 1966) combine several morphological features found in one or other of the two tribes of Sciomyzinae (Sciomyzini and Tetanocerini); thus he doubted a relationship with Tetanocera. Nevertheless, he followed Steyskal's opinion and included Renocera and Anticheta in the Tetanocerini of the Sciomyzinae.

Eight species of Renocera have been described. There are four species in the Nearctic region (Knutson et al., 1986): R. amanda Cresson, 1920; R. brevis (Cresson, $1920)$ (= cyathiformis Melander, 1920); R. johnsoni Cresson, $1920 ; R$. longipes (Loew, 1876) and three in the Palaearctic (Rozkošný, 1987, 1995): R. pallida (Fallén, 1820); R. striata (Meigen, 1830) and R. stroblii Hendel, 1900 .

All Palaearctic species of Renocera are widely distributed throughout Europe including the European part of the former Soviet Union and penetrate to eastern Siberia (R. pallida), Far East of Russia (R. striata) and across Siberia and Mongolia to Kamchatka (R. stroblii).

\section{Minireview of biology of Renocera}

Immature stages have been not described for any Renocera species. However, Foote (1976) noted that long stiff bristles project from the anal region of larvae of $R$. brevis and the anterior spiracles of its puparium are on the tips of long cylindrical tubes, and the last abdominal segment is strongly upturned, and Knutson (1987) presented a figure of the cephalopharyngeal skeleton of Renocera $\mathrm{sp}$. Larvae of Renocera apparently belong to the group of Sciomyzidae known to feed exclusively on clams. The first information on the biology of North American Renocera species was presented by Foote \& Knutson (1970). According to their opinion, the life cycle and larval feeding habits of $R$. brevis are typical of all the reared Nearctic species of Renocera. Adults emerge from overwintering puparia during middle to late May, and the females quickly deposit a hundred or more eggs on shoreline debris close to the water surface. The newly hatched larvae move to the water surface, break through the surface film and crawl or sink to the bottom, where they attack sphaeriid clams of a suitable size. Larvae usually kill the victim immediately after completing the first instar, which lasts from five to nine days. Larvae of the second and third instar kill their prey quickly and consumed it in about an hour. They cannot survive prolonged submergence and always return to the surface film when feeding is completed. Foote (1976) increased our knowledge on many aspects of the biology of North American Renocera spp. The duration in days of the developmental periods of the following species are: $R$. amanda - incubation period 3-5, 1st larval instar 7-10, 2nd instar 8-10, 3 rd instar 24, pupal period not determined. R. brevis incubation period 2-4, 1st larval instar 5-9, 2nd instar
4-7, 3rd instar 8-15, pupal period for male: 11-13, for female: 12-16. R. longipes - incubation period 4-10, 1st larval instar 9-10, 2nd instar 9-10, 3rd instar $10+$ days (the larva died after 10 days), pupal period not determined.

The larvae of all three species kill about 14-25 clams during their development. $R$. brevis can produce two or three generations per year. $R$. amanda and $R$. longipes are univoltine. All three species seemingly overwinter as diapausing pupae within floating or stranded puparia.

Till now only a few records on the biology of Palaearctic species of Renocera have been published. Beaver (1972) noted that the incubation period of the eggs of R. pallida ranged from 6 to 16 days. According to Knutson (1970b) “... some fragmentary observations have shown that Renocera striata Meigen also is a clamkiller".

\section{COLLECTING SITE}

Our laboratory cultures were started using adults collected in the Černovický hájek Nature Reserve ((a fragment of a floodplain forest (Querco-Ulmetum)) near Brno, Czech Republic on 12 May 2000, lgt. J. Horsáková. Flies were swept from herbaceous vegetation mainly composed of Allium ursinum L., Galium aparine L. and Impatiens parviflora DC. The aquatic molluscs encountered at this place, such as Lymnaea stagnalis (Linné, 1758); Galba truncatula (O.F. Müller, 1774); Planorbis planorbis (Linné, 1758); Anisus leucostoma (Millet, 1813); Pisidium subtruncatum Malm, 1855; P. nitidum Jenyns, $1832 ; P$. personatum Malm, 1855 and $P$. casertanum (Poli, 1791) were also collected and offered to the larvae as food.

\section{MATERIAL AND METHODS}

Our laboratory cultures were started with three pairs of adults. Each pair was placed in a separate glass jar over a substrate of damp moss. A mixture of honey and brewer's yeast was supplied as food for the flies. After deposition, eggs were placed in Petri dishes on filter paper moistened daily to determine the duration of the incubation period at room temperature $\left(20-23^{\circ} \mathrm{C}\right)$. Living molluses were added to the Petri dishes immediately after the larvae hatched. The exuviae and some larvae were preserved in 70\% ethanol. First instar larvae were not preserved in order to have a higher number of elder larvae and puparia. Puparia were maintained in a dry state. Preparation for SEM was as follows: after cleaning eggs and larvae were preserved in $70 \%$ ethanol, dehydrated in a graded series of ethanol solutions, dried with $\mathrm{CO}_{2}$ by the critical point technique, and coated with palladium gold before observation under the scanning microscope (TESLA BS 300).

Material examined in this study is deposited in the collection of the author (Brno).

\section{RESULTS}

\section{Morphology of immature stages \\ Egg (Figs 9-12)}

Length $0.75-1.00 \mathrm{~mm}$; greatest width $0.28-0.30 \mathrm{~mm}$. Light brown, elongate oval (Fig. 9). Ventral surface more arched than dorsal surface. Anterior end (Figs 10-11) 


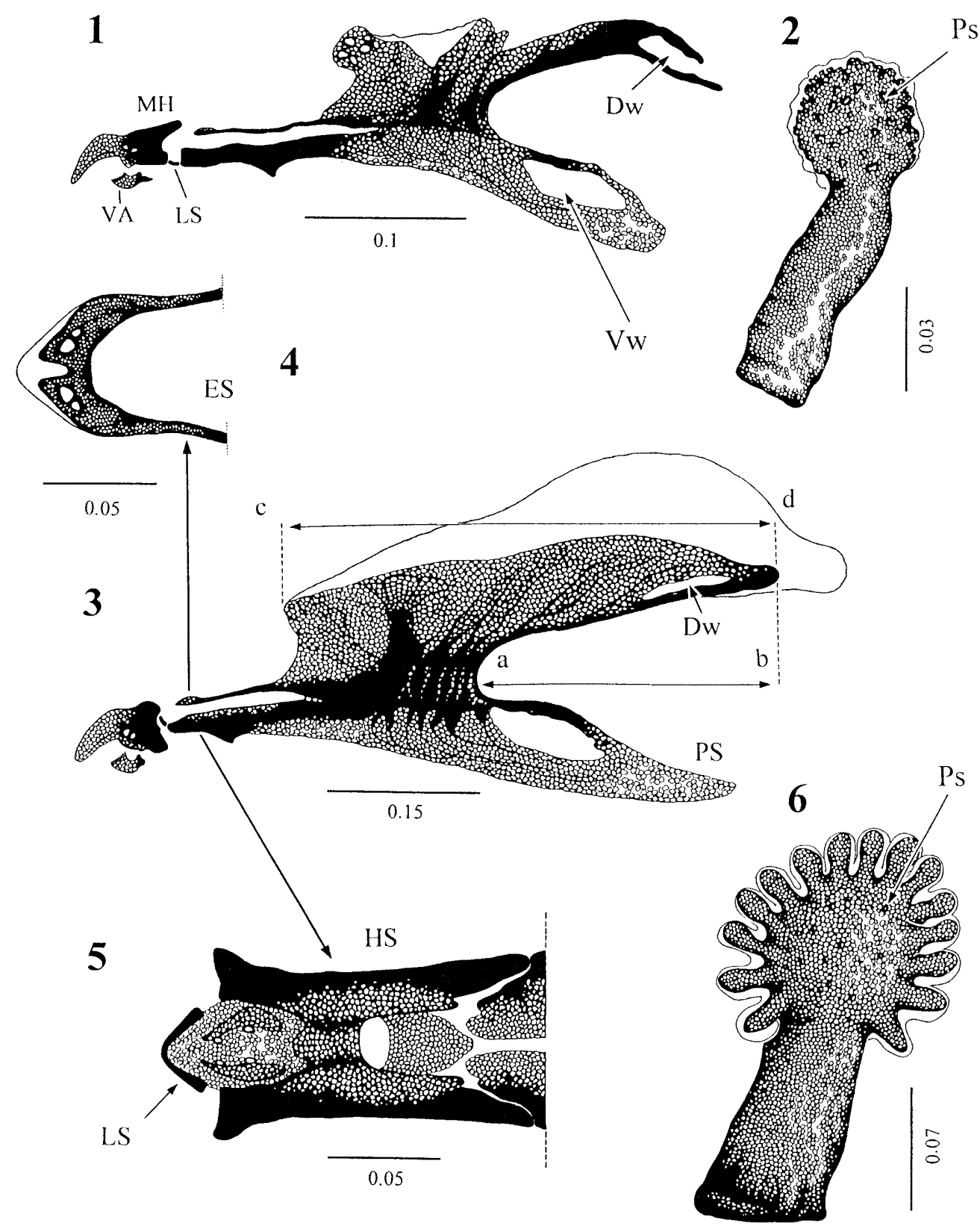

Figs 1-6: Renocera pallida, diagnostic larval characters. 1-2 - second instar larva; 1 - cephalopharyngeal skeleton, MH - mouthhook, VA - ventral arch, LS - ligulate sclerite, Dw - dorsal window, Vw - ventral window; 2 - anterior spiracle, Ps - prismatic spot; 3-6 - third instar larva. 3 - cephalopharyngeal skeleton, PS - pharyngeal sclerite, Dw - dorsal window; 4 - epistomal sclerite (ES); 5 - ligulate sclerite (LS) and hypostomal sclerite (HS); 6 - anterior spiracle, Ps - prismatic spot. All measurements in millimeters.

subconical, with subterminal micropyle (Mp) shielded dorsally by a broad tubercle, bearing apertures. Such apertures are seen only in aquatic and semi-aquatic Sciomyzidae (Gasc et al., 1984). Posterior end (Fig. 12) bulbous with numerous aeropyles (Ae). Chorion sculptured primarily with longitudinal ridges, which, however, are joined by numerous transverse anastomoses. 7 eggs examined.

\section{Second instar larva (Figs 1-2)}

Length $3.45-6.00 \mathrm{~mm}$; greatest width $0.40-0.75 \mathrm{~mm}$. Integument transparent. Thoracic segment I bearing pair of oval anterior spiracles (Fig. 2) 0.095-0.104 mm long, greatest width $0.039-0.044 \mathrm{~mm}$; with distinct prismatic spots (Ps), but without distinct marginal papillae; rudimentary papillae were found only on one larva. Integumental tubercles and creeping welts visible on abdominal segments (though not developed as in most Tetanocerini). Characteristic arrangement of postoral spinule patch formed from indistinct hyaline spines. Pre-anal welt armed with 4-5 transverse rows of low, strong spines the strongest on the surface of the larva. Postanal welt also with spines, however, these are very small and only in 2-3 rows. Posterior spiracular disc surrounded by mas- 


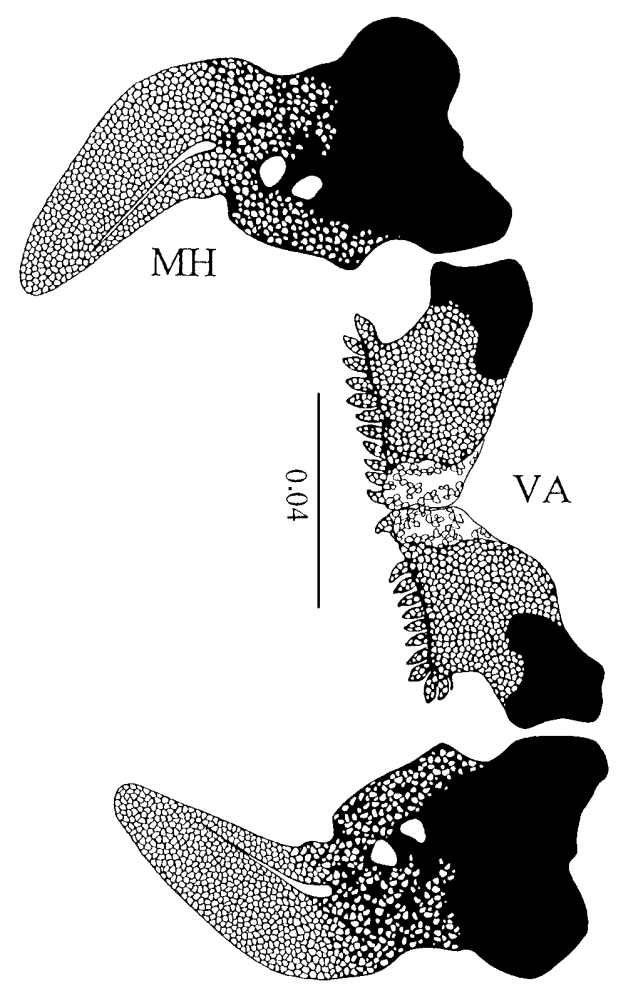

Fig. 7. Renocera pallida, diagnostic larval characters. Mouthhook (MH) and ventral arch (VA). The measurement in millimeters.

sive welt; only ventrolateral lobes well developed. Large dorsal part of marginal welt corresponding to pair of dorsal lobes covered by strong apically darkened spines arranged in 5-7 irregular rows. Ventral part of marginal welt with minute spines located in 7-8 rows. Spiracular disc small, virtually confined to two rounded depressions for protrusion of slender retractile spiracular tubes. Spiracular plates relatively longer than in 3rd instar larvae, with 3 spiracular slits. Cephalopharyngeal skeleton (Fig. 1): length $0.39-0.41 \mathrm{~mm}$; indentation index 60-67. Mouthhooks (MH) with two small apertures, accessory teeth not developed on anterior margin and association with transverse ventral arch not visible. Hook massive, rounded apically, hyaline, little pigmented. Anterior margin of ventral arch (VA) with 22-24 teeth, posterior corners of basal bipartite portion dark. Shape of hypostomal sclerite similar to that in larva of third instar and likewise fused to pharyngeal sclerites. Dorsal cornu of pharyngeal sclerite longer than less sclerotized ventral cornu. One distinct window in each cornu, ventral window $(\mathrm{Vw})$ only slightly wider. 3 larvae examined.

\section{Third instar larva (Figs 3-8, 13-18)}

Length $6.24-7.83 \mathrm{~mm}$; greatest width $1.48-1.76 \mathrm{~mm}$. Integument not pigmented. Body elongate, with strongly tapered and retractile anterior segments consisting of a small cephalic segment and 3 thoracic segments, and less tapered posterior end of 8 abdominal segments. Malpighian tubules visible through middle and posterior parts of body. Cephalic segment mostly rounded, bearing a pair of one-segmented antennae and one pair of ringlike sen-

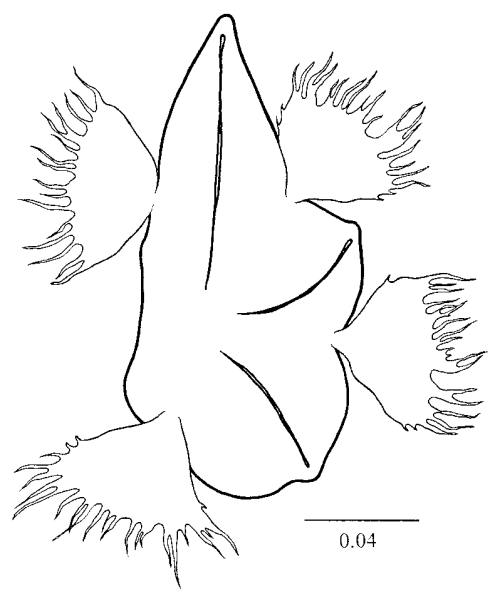

Fig. 8. Renocera pallida, diagnostic larval characters. Spiracular plate. The measurement in millimeters.

silla probably representing maxillary organ. Aperture to atrium on ventral surface. Paired labral sensille papillae on sides of atrium absent. Postoral spinule patch densely covered by small simple spines continuing as longitudinal grooves on posterior half.

With regular lateral tubercles and ventral welts and papillae as in larvae of many Tetanocerini (Rozkošný, 1997, 1998). Thoracic segment I bearing dorsolaterally, on each side, anterior spiracle bordered by 15-17 marginal papillae (Fig. 6). Length of anterior spiracle 0.22 $\mathrm{mm}$, greatest width about $0.13 \mathrm{~mm}$, outer lateral surface with irregular prismatic spots (Ps). Thoracic segments II and III provided with ring of distinct but low tubercles in middle (Fig. 13). Typical integumental structures developed on abdominal segments II-VII. Always two lateral lobes ( $\mathrm{Ll}$ ) present on each side (one above another) of typical abdominal segment, spindle-shaped intersegmental folds (If) developed between segments (Fig. 14). Ventral surface of each abdominal segment divided into three parts (creeping welts) (Fig. 15): Anterior welt (Aw) without papillae, middle welt $(\mathrm{Mw})$ with one pair of papillae (Pa) placed laterally, and posterior welt $(\mathrm{Pw})$ with transverse row of 6 papillae $(\mathrm{Pa})$ arranged in 2 groups on both sides of a mid-median; all 3 sections covered by $4-5$ transverse irregular rows of posteriorly oriented spines, these spines longer and distinctly grouped on top of each papilla.

Posterior spiracular dise with 4 pairs of marginal lobes (Figs 16-17). Only ventrolateral lobes (VLL) elongated; ventral lobe (VL) subtriangular; dorsal (DL) and dorsolateral (DLL) lobes low, tubercle-like. Anal slit situated ventrally in middle of abdominal segment VII. As in second instar larva, pre-anal welt armed with spines; in this case, spines flat, mono- to tricuspidate. Spines on postanal welt indistinct. Virtually entire surface of posterior spiracular disc covered by cuticular spines, only small central area around spiracular tubes smooth. Strongest, darkened cuticular spines on marginal lobes and dorsal area of spiracular disc. True spiracular plates situated apically on slender retractile tubes (St), which are oriented posterodorsally and pointed dorsally, each spi- 

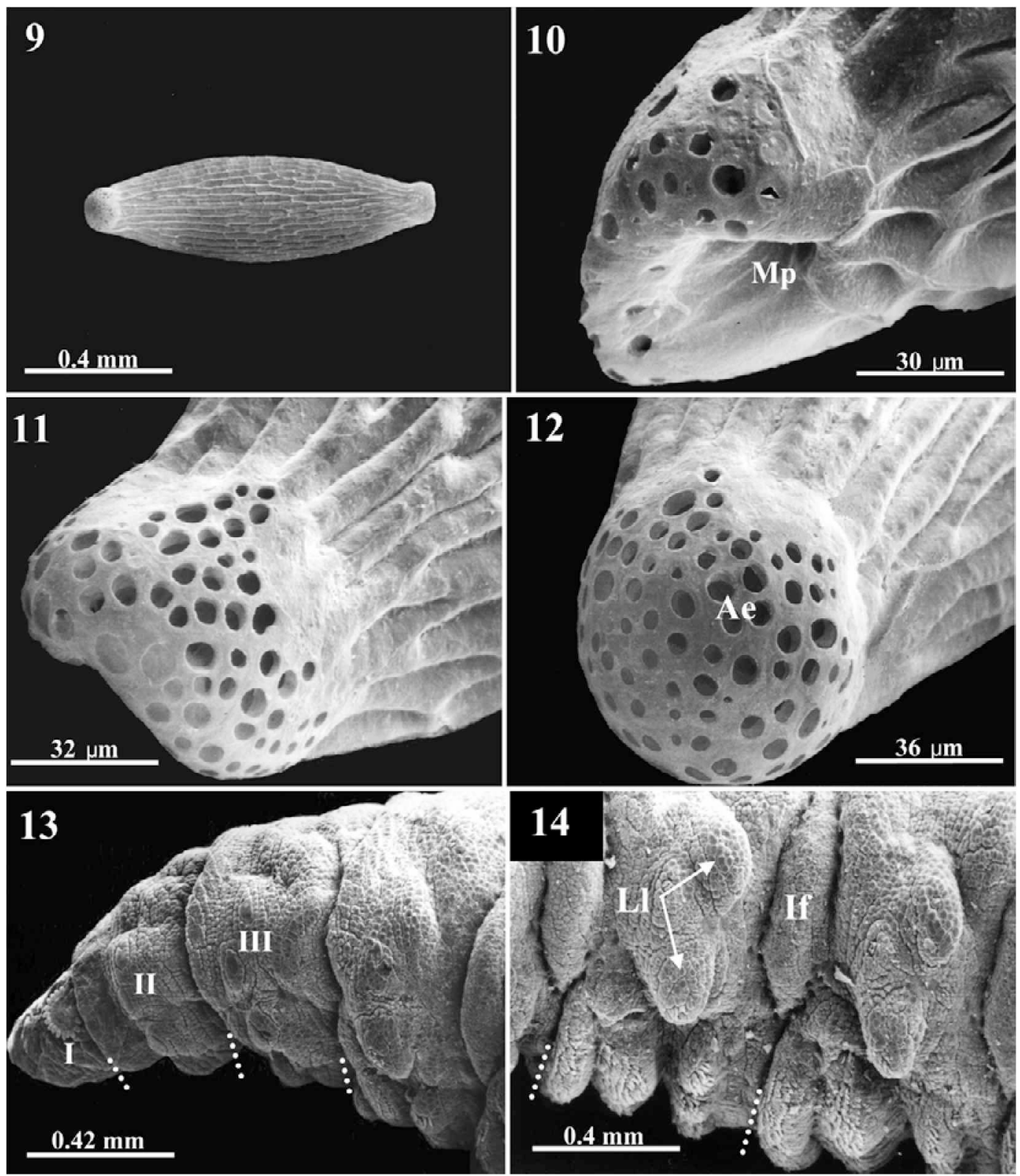

Figs 9-14: Renocera pallida, immature stages. 9-12 - egg; 10-11 - anterior end, $\mathrm{Mp}$ - micropyle; 12 - posterior end, Ae - aeropyles; 13-14 - third instar larva. 13 - thoracic segments I-III, lateral view; 14 - integumental structures on abdominal segment IV, lateral view, L1 - lateral lobes, If - intersegmental fold.

racular plate thus elongated and markedly narrowed towards dorsal tip. Three spiracular slits (Ss, Fig. 18) well developed, spiracular scar in usual position. Due to the unusual tear drop-shape of the spiracular plate, outer spiracular slit is longer than the two other slits (Fig. 8). Four interspiracular processes (Ip) distinct as hyaline plates with marginal fringe (Fig.18). Cephalopharyngeal skeleton (Figs 3-5): length 0.67-0.71 mm; indentation index (ab/cd $\times 100$, Fig. 3) 61-62. Mouthhooks subtriangular, with 2 small windows in basal part; no accesory teeth. Apices of mouthhooks massive and moderately curved but only slightly sclerotized, rounded apically. Ventral arch (Fig. 7) bipartite, with 22 teeth on anterior margin, lateral ends of ventral arch apparently not fused to anteroventral angles of mouthhooks, posterior corners of basal bipartite part darkly pigmented. Transverse epistomal sclerite (ES, Fig. 4) broad, with two pairs of windows and a deep incision in middle anteriorly; epistomal sclerite fused by parastomal bars to pharyngeal sclerites (PS, Fig. 3). Hypostomal sclerite (HS, Fig. 5) U- shaped, totally fused to pharyngeal sclerites; lateral parts of hypostomal sclerite extended dorsally as flange on each side as in other larvae of sciomyzids. Simple ligulate sclerite (LS, Fig. 5) and lightly pigmented sensory plate with 2 pairs of apertures between anterior arms of hypostomal sclerite joined by lightly pigmented connections. Pharyngeal sclerites (PS, Fig. 3) not connected by a dorsal bridge. Dorsal cornu only slightly longer than ventral cornu; dorsal window (Dw) distinct, but very narrow, 

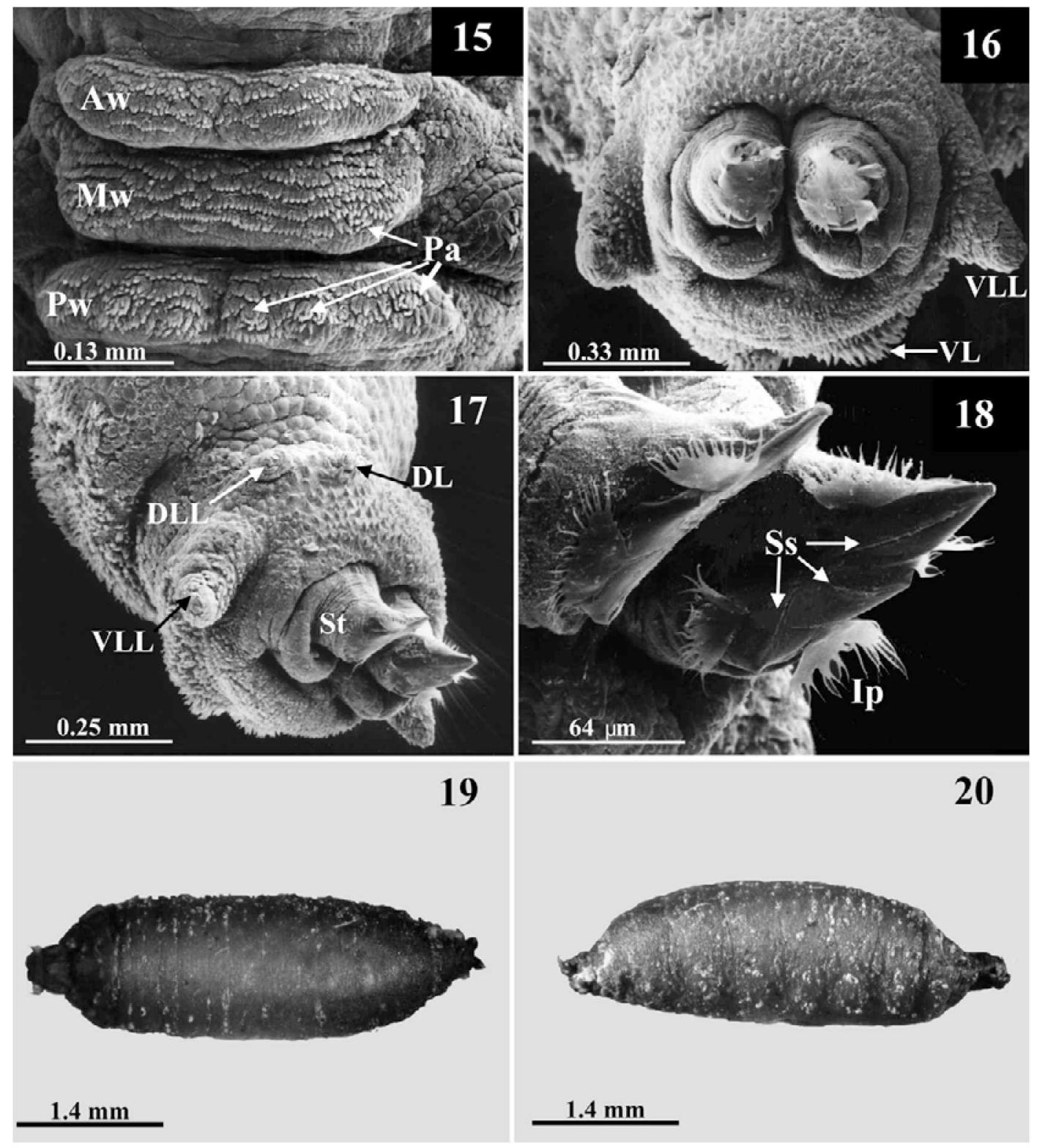

Figs 15-20: Renocera pallida, immature stages. 15-18 - third instar larva; 15 - creeping welts on abdominal segment II, ventral view, $\mathrm{Aw}$ - anterior welt, $\mathrm{Mw}$ - middle welt, $\mathrm{Pw}$ - posterior welt, $\mathrm{Pa}$ - papillae; 16-17 - posterior spiracular disc, $\mathrm{DL}$ - dorsal lobe, DLL - dorsolateral lobe, VL - ventral lobe, VLL - ventrolateral lobe; 18 - spiracular plate, Ss - spiracular slits, Ip - interspiracular process; 19-20 - puparium, dorsal and lateral view.

window in ventral cornu oval, elongate. 3 larvae examined.

\section{Puparium (Figs 19-20)}

Length $4.10-4.30 \mathrm{~mm}$; greatest width $1.60-1.70 \mathrm{~mm}$. General shape conspicuously elongate, rather cylindrical in comparison with other, mostly barrel-shaped puparia of the family. Both ends abruptly narrowed, dorsal surface more arched than ventral surface. Colour reddish-brown, both ends darkened. Narrow anterior end with distinct median incision, vestiges of anterior spiracles are present in anterior corners as distinct rounded vertical plates with about 16 marginal papillae. Thoracic and abdominal segments distinct, especially on lateral surfaces of abdomen where intersegmental folds are distinct. Secondary annu- lation maintained, especially on ventral surface of abdominal segments where ventral spinule patches are visible as transverse stripes of pale pubescence (in lateroventral view). Posterior end of puparium directed almost horizontally, although closer to ventral surface. Spiracular tubes prominent on posterior spiracular area, their terminal plates oriented posteriorly and slightly laterally. As in larvae, spiracular tubes conspicuously pointed dorso-apically. Vestiges of lateral lobes around spiracular field just visible, vestiges of ventrolateral lobes protuberant. Peri-anal welt low, bordering a small, transverse oval cavity in place of anal slit of larva. 2 puparia examined. 


\section{Biology}

R. pallida, the most common species of the genus in the Palaearctic, occurs mostly at lower altitudes in moist meadows and marshes. In Central Europe, adults are present from May to September. In the laboratory, only one female, collected on 12 May, laid eggs and produced total of 25 by 14 May. Eggs were deposited individually on the walls of the rearing jar, moss and vegetation, but never on the shells of living sphaeriid clams. In one case a chainlike arrangement of 5 eggs was observed on the margin of a blade of Typha sp. The incubation period was 3-4 days at room temperature. After hatching, the larvae were placed in a large dish of water, about $8 \mathrm{~cm}$ deep, containing living aquatic molluscs (see Collecting sites), including two species of fingernail clams: Sphaerium corneum (Linné, 1758) and Musculium lacustre (O.F. Müller, 1774). However, the larvae were not able to sink to the bottom and reach the snails and clams. On the contrary they tried to crawl onto the floating plants and leave the water. In the second experiment the larvae were placed in Petri dishes with moist filter paper or little water in which the clams were submerged. Such conditions simulate the semi-aquatic habitats (marshes and moist upper soil layers in floodplain forests) where some species of Pisidium C. Pfeifer, 1821 and especially $P$. personatum are found. In this experiment the larvae did not attack the freshwater snails but attacked only the sphaeriid clams. First-instar larvae preferred small specimens of the genus Pisidium. In addition, older larvae also ate larger species of Sphaerium Scopoli, 1777 and Musculium Link, 1807. When a suitable clam was encountered, the 1st instar larva slowly forced its way between the slightly gaping valves and entered the mantle cavity. Contact with the air was maintained by the pair of posterior spiracles, which were macroscopically distinct as two dark points between the edges of the slightly opened shells. Larvae usually killed the clam immediately after completing the 1st larval instar $(\mathrm{n}=5)$, which lasted about 5 days. Feeding was restricted to the softer tissues; the foot was rarely eaten. Larvae of the 2 nd instar were able to extend their slender retractile spiracular tubes upwards through the gap between valves. The apically placed and dorsally pointed spiracular plates were distinct as two pale horns. Later, when larvae were more mature, only the anterior half of their body was usually wedged in the clam during feeding. The feeding behaviour was different from that of the first instar. In a few hours they consumed the entire contents of the shell and then rested on the moist filter paper for a short time. The second stadium $(n=5)$ lasted 6 days. Third instar larvae killed clams very quickly and remained within the prey for about an hour. In pauses between feeding they rested on the margins of the Petri dishes for a day. They were able to kill and eat at most two specimens of Pisidium per day. After completing the 3rd larval instar, which took from 19 to 20 days $(\mathbf{n}=2)$, two larvae formed puparia outside the shells of the clams. Only one puparium produced an adult, a male after 43 days.

\section{DISCUSSION}

The morphological and behavioural data on the immature stages of $R$. pallida presented here confirm the generic characteristics given by Foote (1976) for Nearctic species. The eggs and larvae of $R$. pallida, as those of Anticheta, cannot be unambiguously classified as belonging to either the Sciomyzini or Tetanocerini. Their immature stages show morphological features typical of one or the other tribe or combinations of both. There are two types of eggs in the Sciomyzidae based on their chorion structure, but distinguishing tribes on the basis of this character is not reliable. Longitudinally ridged eggs are characteristic of most genera of Tetanocerini but not Anticheta, the chorion of which consists of almost hexagonal fields typical of the Sciomyzini (with some exceptions, Euthycera Latreille, 1829; Sepedon) (Rozkošný, 1998). In contrast, Pherbellia cinerella (Fallén, 1820), with longitudinally furrowed eggs, is an exception among the Sciomyzini (Bratt et al., 1969). The primary surface structure of $R$. pallida eggs resembles that of the Tetanocerini but the secondary structure consists of elongate fields formed by anastomoses between longitudinal ridges. There are two morphologically distinct type of larvae in the Sciomyzinae. Larvae of Sciomyzini and Anticheta have a relatively smooth integument and movement is aided by means of transverse spinule patches or bands. The body segments of free living larvae of most Tetanocerini are provided with numerous folds, groups of tubercles, papillae, and ventral creeping welts. The intermediate larvae of $R$. pallida have, in addition to all of the main surface structures typical of the Tetanocerini, ventral spinule patches consisting of irregular rows of cuticular spines on all ventral transverse welts on abdominal segments II-VII. Their posterior spiracular disc is surrounded by weakly developed marginal lobes (except for the ventrolateral lobes), as in the larvae of Sciomyzini and terrestrial Tetanocerini. The form of the dorso-apically pointed, retractile, slender, spiracular tubes is especially unique. Presence of interspiracular processes is not a diagnostic criterion for the systematic position of Renocera because SEM studies by Vala \& Gasc (1990) have revealed the presence of these structures in larvae of Pherbellia cinerella, $P$. dorsata (Zetterstedt, 1846) and $P$. schoenherri, where they were presumed to be absent (cf. Bratt et al., 1969), and Rozkošný \& Knutson (1970) found them in first instar larvae of 3 species of Pteromicra (Sciomyzini). Combinations of characters of the cephalopharyngeal skeleton of Sciomyzini and Tetanocerini are present along with atypical structures. Accessory teeth on the mouthhooks (characteristic of Tetanocerini) are absent as is the ventral arch - fusion of mouthhooks described by Knutson (1966) for most larvae of Anticheta and by Knutson (1970a) for Tetanura pallidiventris Fallén, 1820 (Sciomyzini). The shape of the mouthhooks seems to be unique, with the apices massive but only slightly pigmented. The fusion of the hypostomal sclerite with the pharyngeal sclerites found in mature larvae of Renocera and Anticheta may, at most, be interpreted as evidence of a relationship between Renocera 
and Anticheta. In contrast to the Sciomyzini and Anticheta, but similar to the Tetanocerini, the dorsal bridge of $R$. pallida is virtually absent and the window in the dorsal cornu of the pharyngeal sclerites is reduced. On the other hand, the value of the indentation index is close to that of the Sciomyzini (60-67).

Compared with $R$. pallida, the other two known clamkilling flies, New Zealand Eulimnia philpotti and Palaearctic Ilione lineata, have a combination of morphological features characteristic of the Tetanocerini: egg chorion striate, larvae with accessory teeth on the mouthhooks, 3rd instar indentation index less than 50, long lobes around the posterior spiracular disc in aquatic species; only one window in the cornu (ventral), always without a dorsal bridge between the pharyngeal sclerites, and absence of ventral spinule patches (Knutson \& Berg, 1967; Barnes, 1980).

The biology of $R$. pallida is generally very similar to that of the 3 Nearctic species. First instar larvae show distinct parasitoid tendencies and only kill their host after a few days of feeding. Second and 3rd instar larvae are typical predators. The duration of the complete life cycle is very similar to that of the Nearctic species; the data for $R$. pallida may be summarized as follows: the complete life cycle requires $76-78$ days (excluding premating and pre-ovoposition periods), thus at most they complete two generations per a year and very probably overwinter as puparia. These conclusions are similar to those of Berg et al. (1982) who placed the genus Renocera in their phenological group 1 (multivoltine species that overwinter as pupae).

The larvae do not show any adaptations for a submerged mode of life. On the basis of the above experiments they probably attack sphaeriid clams living in semi-aquatic conditions (edges of aquatic habitats). The slender, retractile, unusually shaped and dorso-apically pointed spiracular tubes on the posterior spiracular disc are an adaptation to breathing through the fissure between the valves of the shell.

ACKNOWLEDGEMENTS. I would like to thank Prof. R. Rozkošny for his help with many aspect of this study. I am also very obliged to Dr. L. V. Knutson and Prof. J.-C. Vala for critical comments on manuscript, my husband M. Horsák for determining the molluses, and M. Kudrna for taking the photographs. This research was supported by grant MSM 143100010.

\section{REFERENCES}

BARNES J.K. 1980: Taxonomy of the New Zealand genus Eulimnia, and biology and immature stages of E. philpotti (Diptera: Sciomyzidae). N. Z. J. Zool. 7: 91-103.

BeAver O. 1972: Notes on the biology of some British sciomyzid flies (Diptera: Sciomyzidae). II. Tribe Tetanocerini. Entomologist (London) 105: 284-299.

BERG C.O. 1953: Sciomyzid larvae (Diptera) that feed on snails. J. Parasitol. 39: 630-636.

Berg C.O., Foote B.A., Knutson L., Barnes J.K., Arnold S.L. \& Valley K. 1982: Adaptive differences in phenology in sciomyzid flies. Mem. Entomol. Soc. Wash. 10: 15-36.

Berg C.O. \& Knutson L. 1978: Biology and systematics of the Sciomyzidae. Ann. Rev. Entomol. 23: 239-258.
Bratt A.D., Knutson L.V., Foote B.A. \& Berg C.O. 1969: Biology of Pherbellia (Diptera: Sciomyzidae). N. Y. Agric. Exp. Stn. Ithaca Mem. 404: 1-246.

Coupland J.B., Espiau A. \& BaKer G. 1994: Seasonality, longevity, host choice, and infection efficiency of Salticella fasciata (Diptera: Sciomyzidae), a candidate for the biological control of pest helicid snails. Biol. Cont. 4: 32-37.

Fisher T.W. \& ORTH R.E. 1964: Biology and immature stages of Antichaeta testacea Melander (Diptera: Sciomyzidae). Hilgardia 36: 1-29.

Foote B.A. 1976: Biology and larval feeding habits of three species of Renocera (Diptera: Sciomyzidae) that prey on fingernail clams (Mollusca: Sphaeriidae). Ann. Entomol. Soc. Am. 69: 121-133.

Foote B.A. \& KnUtson L.V. 1970: Clam-killing fly larvae. Nature (London) 226(5244): 466.

Gasc C., Vala J.-C. \& Redienbach J.M. 1984: Étude comparative au microscope électronique a balayage des structures chorioniques d'oeufs de cinq espčces de Sciomyzidae a larves terrestres et aquatiques (Diptera). Annls Soc. Entomol. Fr. (N. S.) 20: $163-170$.

KnUtSon L.V. 1966: Biology and immature stages of malacophagous flies: Antichaeta analis, A. atriseta, A. brevipennis, and A. obliviosa (Diptera: Sciomyzidae). Trans. Amer. Entomol. Soc. 92: 67-101.

KNUTSON L.V. 1970a: Biology and immature stages of Tetanura pallidiventris, a parasitoid of terrestrial snails (Dipt.: Sciomyzidae). Entomol. Scand. 1: 81-89.

KNUTSON L.V. 1970b: Biology of snail-killing flies in Sweden (Dipt., Sciomyzidae). Entomol. Scand. 1: 307-314.

KNUTSOn L. 1987: 84. Family Sciomyzidae. In: McAlpine J.F. (ed.): Manual of Nearctic Diptera. Vol. 2. Res. Br. Agric. Canada 28: 927-940.

KNUTSON L. \& ABERCROMBIE J. 1977: Biology of Antichaeta melanosoma (Diptera: Sciomyzidae), with notes on parasitoid Braconidae and Ichneumonidae (Hymenoptera). Proc. Entomol. Soc. Washington 79: 111-125.

KNutson L.V. \& Berg C.O. 1967: Biology and immature stages of malacophagous Diptera of the genus Knutsonia Verbeke (Sciomyzidae). Bull. Inst. Sci. Nat. Belg. 43: 1-60.

Knutson L., Orth R.E., Fisher T.W. \& Murphy W.L. 1986: Catalog of Sciomyzidae (Diptera) of America North of Mexico. Entomography 4: 1-52.

Knutson L.V., RozkošnÝ R. \& Berg C.O. 1975: Biology and immature stages of Pherbina and Psacadina (Diptera: Sciomyzidae). Acta Sc. Nat. Brno 9: 1-38.

Knutson L.V., Stephenson J.W. \& Berg C.O. 1965: Biology of a slug-killing fly, Tetanocera elata (Diptera: Sciomyzidae). Proc. Malac. Soc. Lond. 36: 213-220.

Knutson L.V., S SePHenson J.W. \& Berg C.O. 1970: Biosystematic studies of Salticella fasciata (Meigen), a snailkilling fly (Diptera: Sciomyzidae). Trans. R. Entomol. Soc. Lond. 122: 81-100.

Marinoni L. \& Mathis W.N. 2000: A cladistic analysis of Sciomyzidae Fallén (Diptera). Proc. Biol. Soc. Washington 113: 162-209.

Robinson W.H. \& Foote B.A. 1978: Biology and immature stages of Antichaeta borealis (Diptera: Sciomyzidae), a predator of snail eggs. Proc. Entomol. Soc. Washington 80: 388-396.

RozkošnÝ R. 1987: A Review of the Palaearctic Sciomyzidae (Diptera). Univerzita J.E. Purkyne, Brno, 100 pp., 56 pls.

RozKoŠNÝ R. 1995: World distribution of Sciomyzidae based on the list of species (Diptera). Studia Dipterologica 2: 221-238. 
RoZKoŠNÝ R. 1997: Diptera Sciomyzidae, snail killing flies. In Nilsson A.N. (ed.): Aquatic Insects of North Europe - A Taxonomic Handbook. Apollo Books, Stenstrup, pp. 363-381.

RozkoŠnÝ R. 1998: Family Sciomyzidae. In: Papp L. \& Darvas B. (eds): Contributions to Manual of Palaearctic Diptera (with Special Reference to Flies of Economic Importance). Vol. 3. Higher Brachycera. Science Herald, Budapest, pp. 357-382.

RozKošnÝ R. \& KNutson L.V. 1970: Taxonomy, biology, and immature stages of Palearctic Pteromicra, snail-killing Diptera (Sciomyzidae). Ann. Entomol. Soc. Am. 63: 1434-1459.

StEYSKal G.C. 1965: The subfamilies of Sciomyzidae of the world. (Diptera: Acalyptratae). Ann. Entomol. Soc. Am. 58: 593-594.

TrelKa D.G. \& Foote B.A. 1970: Biology of slug-killing Tetanocera (Diptera: Sciomyzidae). Ann. Entomol. Soc. Am. 63: $877-895$.
VAla J.-C. \& Gasc C. 1990: Ecological adaptations and morphological variation in the posterior disc of larvae of Sciomyzidae (Diptera). Can. J. Zool. 68: 517-521.

Vala J.-C., Gbedissi G., Knutson L.V. \& Dossou C. 2000: Extraordinary feeding behaviour in Diptera Sciomyzidae, snail-killing flies. C. R. Acad. Sci. Paris, Sciences de la Vie/ Life Sciences 323: 299-304.

Verbere J. 1950: Fasc. 66. Sciomyzidae (Diptera: Cyclorrhapha). Exploration Parc Nat. Albert, Mission de Witte (1933-35), Brussels, 97 pp.

Verbere J. \& Knutson L.V. 1967: Sciomyzidae. In: Illies J. (ed.): Limnofauna Europaea. Gustav Fischer Verlag, Stuttgart, pp. 485-488.

Received December 5, 2001; revised July 25, 2002; accepted November 14, 2002 